\title{
PRESCRIBING THE PRESCHWARZIAN IN SEVERAL COMPLEX VARIABLES
}

\author{
Rodrigo Hernández \\ Universidad Adolfo Ibáñez, Facultad de Ciencias y Tecnología \\ Av. Balmaceda 1625 Recreo, Viña del Mar, Chile; rodrigo.hernandez@uai.cl
}

\begin{abstract}
We solve the several complex variables preSchwarzian operator equation $[D f(z)]^{-1}$ $D^{2} f(z)=A(z), z \in \mathbf{C}^{n}$, where $A(z)$ is a bilinear operator and $f$ is a $\mathbf{C}^{n}$ valued locally biholomorphic function on a domain in $\mathbf{C}^{n}$. Then one can define a several variables $f \rightarrow f_{\alpha}$ transform via the operator equation $\left[D f_{\alpha}(z)\right]^{-1} D^{2} f_{\alpha}(z)=\alpha[D f(z)]^{-1} D^{2} f(z)$, and thereby, study properties of $f_{\alpha}$. This is a natural generalization of the one variable operator $f_{\alpha}(z)$ in [6] and the study of its univalence properties, e.g., the work of Royster [23] and many others. Möbius invariance and the multivariables Schwarzian derivative operator of Oda [17] play a central role in this work.
\end{abstract}

\section{Introduction}

Consider the class $\mathscr{S}$ of functions $f$ holomorphic and univalent in the disk $\mathbf{D}=$ $\{z:|z|<1\}$ with the normalization $f(0)=0$ and $f^{\prime}(0)=1$. Let $\alpha \in \mathbf{C}, f \in \mathscr{S}$ and define the integral transform

$$
f_{\alpha}(z)=\int_{0}^{z}\left[f^{\prime}(w)\right]^{\alpha} d w
$$

where the power is defined by the branch of the logarithm for which $\log f^{\prime}(0)=0$, [6]. A question considered in [6] is to determinate the values of $\alpha$ for which $f_{\alpha} \in \mathscr{S}$. In [23] Royster exhibited non-univalent mappings $f_{\alpha}$ for each complex $\alpha \neq 1$ with $|\alpha|>1 / 3$. In fact, consider functions of the form

$$
f(z)=\exp (\mu \log (1-z)),
$$

which are univalent if and only if $\mu$ lies in ones of the closed disks

$$
|\mu+1| \leq 1, \quad|\mu-1| \leq 1 .
$$

Royster showed that for any such value of $\mu$, the function in (1.1) is not univalent for each $\alpha$ with $|\alpha|>1 / 3$ and $\alpha \neq 1$. Moreover, Pfaltzgraff using the Ahlfors univalence criterion [1] proved that for any $f \in \mathscr{S}$, if $|\alpha| \leq 1 / 4$, then $f_{\alpha}$ is univalent in $\mathbf{D}$, see [19].

Let $f$ be a locally univalent mapping in $\mathbf{D}$ and $f_{\alpha}$ defined by equation (1.1). Then $f_{\alpha}^{\prime}(z)=\left[f^{\prime}(z)\right]^{\alpha}$, which implies that

$$
\frac{f_{\alpha}^{\prime \prime}}{f_{\alpha}^{\prime}}(z)=\alpha \frac{f^{\prime \prime}}{f^{\prime}}(z) .
$$

doi:10.5186/aasfm.2011.3621

2010 Mathematics Subject Classification: Primary 32H02, 32W99; Secondary 32A10.

Key words: PreSchwarzian derivative, holomorphic mapping, univalence.

The author was partially supported by Fondecyt Grants \# 11070055. 
If $f$ and $g$ satisfy that $g^{\prime \prime} / g^{\prime}(z)=f^{\prime \prime} / f^{\prime}(z)$, then $\log \left(g^{\prime}(z)\right)=\log \left(f^{\prime}(z)\right)$ when $f^{\prime}(0)=$ $g^{\prime}(0)$. Therefore $g=f$ if $f(0)=g(0)$. Thus

$$
f_{\alpha}(z)=\int_{0}^{z}\left[f^{\prime}(w)\right]^{\alpha} d w \Leftrightarrow \frac{f_{\alpha}^{\prime \prime}}{f_{\alpha}^{\prime}}(z)=\alpha \frac{f^{\prime \prime}}{f^{\prime}}(z) .
$$

This equivalence in one variable suggests our idea to define the several variables generalization of $f_{\alpha}$ via the operator equation

$$
\left[D f_{\alpha}(z)\right]^{-1} D^{2} f_{\alpha}(z)(\cdot, \cdot)=\alpha[D f(z)]^{-1} D^{2} f(z)(\cdot, \cdot) .
$$

Yoshida [25] developed a complete description of prescribing Oda's Schwarzian derivatives [17] in terms of a completely integrable system of differential equations. The description involves operators $S_{i j}^{k} f$ and $S_{i j}^{0} f$ of orders two and three respectively, coefficients of the system and Möbius invariants. In fact, the $S_{i j}^{k} f$ operators are the operator of least order that vanish for Möbius mappings. This is a strong difference with one complex variable where the third order Schwarzian operator is the lowest order operator annihilated by all Möbius mappings. For $n=1$, the Möbius group has dimension 3 , which allows to set $f\left(z_{0}\right), f^{\prime}\left(z_{0}\right)$ and $f^{\prime \prime}\left(z_{0}\right)$ of a holomorphic mapping $f$ at a given point $z_{0}$ arbitrarily. It would therefore be pointless to seek a Möbius invariant differential operator of order 2. But for $n>1$ the number of parameters involved in the value and all derivatives of order 1 and 2 of a locally biholomorphic mapping is $n^{2}(n+1) / 2+n^{2}+n$, and exceeds the dimension of the corresponding Möbius group in $\mathbf{C}^{n}$, which is $n^{2}+2 n$. By the definition of the Schwarzian derivatives, we have that $S_{i j}^{k} F=S_{j i}^{k} F$ for all $k$ and $\sum_{j=1}^{n} S_{i j}^{j} F=0$ and we see there are exactly $n(n-1)(n+2) / 2$ independent terms $S_{i j}^{k} F$, which is equal to the excess mentioned above.

A different approach to obtain the invariant operators $S_{i j}^{k}, S_{i j}^{0}$ has been developed by Molzon and Tamanoi [14]. In addition, Molzon and Pinney had earlier developed equivalent invariant operators in the context of complex manifolds [13].

The operator

$$
P_{f}(z)=[D f(z)]^{-1} D^{2} f(z)(\cdot, \cdot)
$$

introduced by Pfaltzgraff in [18] is the "natural" way to extend the classical one variable operator preSchwarzian $f^{\prime \prime} / f^{\prime}$. Furthermore, the author in [18] extended the classical univalence criterion of Becker [2] to several variables. The question now is how to extend the equation (1.1) to $\mathbf{C}^{n}$. It is necessary to understand when one can recover the function $f$ from a given $P_{f}$. We shall show a strong connection between this operator and the Schwarzian derivatives operator $S F(z)(\cdot, \cdot)$, introduced in [11]. Indeed, the problem of prescribing $P_{f}$ can be reduced to understanding how to prescribe $S_{i j}^{k} f$ in terms of $P_{f}$. This is achieved via completely integrable system generated by $S_{i j}^{k} f$ and corresponding "new differential conditions" on the elements of $P_{f}$. We then use this theory to extend the classical single variable problem about the univalence of $f_{\alpha}$ by using equation (1.4) to define $f_{\alpha}$ in several complex variables.

\section{Oda Schwarzian and Möbius invariants}

Let $f: \Omega \subset \mathbf{C}^{n} \rightarrow \mathbf{C}^{n}$ be a locally biholomorphic mapping defined on some domain $\Omega$. Oda in [17] defined the Schwarzian derivatives of $f=\left(f_{1}, \ldots, f_{n}\right)$ as 


$$
S_{i j}^{k} f=\sum_{l=1}^{n} \frac{\partial^{2} f_{l}}{\partial z_{i} \partial z_{j}} \frac{\partial z_{k}}{\partial f_{l}}-\frac{1}{n+1}\left(\delta_{i}^{k} \frac{\partial}{\partial z_{j}}+\delta_{j}^{k} \frac{\partial}{\partial z_{i}}\right) \log J_{f}
$$

where $i, j, k=1,2, \ldots, n, J_{f}$ is the jacobian determinant of the differential $D f$ and $\delta_{i}^{k}$ are the Kronecker symbols. For $n>1$ the Schwarzian derivatives have the following properties:

$$
S_{i j}^{k} f=0 \text { for all } i, j, k=1,2, \ldots, n \text { iff } f(z)=M(z)
$$

for some Möbius transformation

$$
M(z)=\left(\frac{l_{1}(z)}{l_{0}(z)}, \ldots, \frac{l_{n}(z)}{l_{0}(z)}\right),
$$

where $l_{i}(z)=a_{i 0}+a_{i 1} z_{1}+\cdots+a_{i n} z_{n}$ with $\operatorname{det}\left(a_{i j}\right) \neq 0$. Furthermore, for a composition

$$
S_{i j}^{k}(g \circ f)(z)=S_{i j}^{k} f(z)+\sum_{l, m, r=1}^{n} S_{l m}^{r} g(w) \frac{\partial w_{l}}{\partial z_{i}} \frac{\partial w_{m}}{\partial z_{j}} \frac{\partial z_{k}}{\partial w_{r}}, w=f(z) .
$$

From this chain rule it can be shown that $S_{i j}^{k} f=S_{i j}^{k} g$ for all $i, j, k=1, \ldots, n$ if and only if $g=T \circ f$ for some Möbius transformation. The $S_{i j}^{0} f$ coefficients are given by

$$
S_{i j}^{0} f(z)=J_{f}^{1 /(n+1)}\left(\frac{\partial^{2}}{\partial z_{i} \partial z_{j}} J_{f}^{-1 /(n+1)}-\sum_{k=1}^{n} \frac{\partial}{\partial z_{k}} J_{f}^{-1 /(n+1)} S_{i j}^{k} f(z)\right) .
$$

In his work, Oda gives a description of the functions with prescribed Schwarzian derivatives $S_{i j}^{k} f([17])$. Consider the following overdetermined system of partial differential equations,

$$
\frac{\partial^{2} u}{\partial z_{i} \partial z_{j}}=\sum_{k=1}^{n} P_{i j}^{k}(z) \frac{\partial u}{\partial z_{k}}+P_{i j}^{0}(z) u, \quad i, j=1,2, \ldots, n,
$$

where $z=\left(z_{1}, z_{2}, \ldots, z_{n}\right) \in \Omega \subset \mathbf{C}^{n}$ and $P_{i j}^{k}(z)$ are holomorphic functions for $i, j, k=$ $0, \ldots, n$. The system $(2.4)$ is called completely integrable if there are at most $n+1$ linearly independent solutions, and is said to be in canonical form (see [24]) if the coefficients satisfy

$$
\sum_{j=1}^{n} P_{i j}^{j}(z)=0, \quad i=1,2, \ldots, n .
$$

Oda proved that (2.4) is a completely integrable system in canonical form if and only if $P_{i j}^{k}=S_{i j}^{k} f$ for a locally biholomorphic mapping $f=\left(f_{1}, \ldots, f_{n}\right)$, where $f_{i}=u_{i} / u_{0}$ for $1 \leq i \leq n$ and $u_{0}, u_{1}, \ldots, u_{n}$ is a set of linearly independent solutions of the system. For a given mapping $f, u=\left(J_{f}\right)^{-\frac{1}{n+1}}$ is always a solution of $(2.4)$ with $P_{i j}^{k}=S_{i j}^{k} f$.

Definition 2.1. We define the Schwarzian derivative operator as the operator $S_{f}(z): T_{z} \Omega \rightarrow T_{f(z)} \Omega$ given by

$$
S_{f}(z)(\vec{v}, \vec{w})=\left(\vec{v}^{t} S^{1} f(z) \vec{w}, \ldots, \vec{v}^{t} S^{n} f(z) \vec{w}\right),
$$

where $S^{k} f$ is the $n \times n$ matrix defined by $\left(S_{i j}^{k} f\right)_{i j}$ and $\vec{v} \in T_{z} \Omega$. 
The Schwarzian derivative operator [12] can be rewritten as

$$
\begin{aligned}
S_{f}(z)(\vec{v}, \vec{w})= & {[D f(z)]^{-1} D^{2} f(z)(\vec{v}, \vec{w})-\frac{1}{n+1}\left(\nabla \log J_{f}(z) \cdot \vec{v}\right) \vec{w} } \\
& -\frac{1}{n+1}\left(\nabla \log J_{f}(z) \cdot \vec{w}\right) \vec{v},
\end{aligned}
$$

and the system $(2.4)$ as

$$
\operatorname{Hess} u(z)(\cdot, \cdot)=\nabla u(z) \cdot S_{f}(z)(\cdot, \cdot)+S_{f}^{0}(z)(\cdot, \cdot) u(z),
$$

where $S_{f}^{0}$ is a $n \times n$ matrix defined by $\left(S_{i j}^{0} f\right)_{i j}$. We include in this section two lemmas that complement the work of Oda.

Lemma 2.2. Let $f: \Omega \subset \mathbf{C}^{n} \rightarrow \mathbf{C}^{n}$ be a locally biholomorphic mapping and $u_{0}=J_{f}^{-1 / n+1}$. Then

$$
f=\frac{\vec{u}}{u_{0}}=\left(\frac{u_{1}}{u_{0}}, \ldots, \frac{u_{n}}{u_{0}}\right),
$$

where $u_{0}, u_{1}, \ldots, u_{n}$ are linearly independent solutions of (2.4)

Proof. We will prove that $\vec{u}=f u_{0}$ is solution of the equation (2.6). It follows that $D f u_{0}+f \nabla u_{0}=D u$, from where

$$
D^{2} f \cdot u_{0}+2 D f \cdot \nabla u_{0}+f \cdot \operatorname{Hess} u_{0}=D^{2} u \text {. }
$$

Using the system we have that

$$
D^{2} f \cdot u_{0}+2 D f \cdot \nabla u_{0}-D f \cdot u_{0} \cdot S_{f}+D u \cdot S_{f}+S_{f}^{0} \cdot u=D^{2} u .
$$

Considering the equation $(2.5)$ with $u_{0}=J_{f}^{-1 / n+1}$ we have that

$$
D^{2} f \cdot u_{0}+2 D f \cdot \nabla u_{0}-D f \cdot S f \cdot u_{0}=0,
$$

and $D^{2} u(\cdot, \cdot)=D u\left(S_{f}(\cdot, \cdot)\right)+S_{f}^{0}(\cdot, \cdot) u$, hence $u_{i}$ with $i=1, \ldots, n$ and $u_{0}$ are independent solutions of the system (2.4).

Lemma 2.3. Let $u_{0}$ be a solution of the system (2.4). Then there exists a function $f=\vec{u} / u_{0}$ where $\vec{u}=\left(u_{1}, \ldots, u_{n}\right)$ and $u_{i}$ with $i=0,1, \ldots, n$ are independent solutions of the system (2.4) where $u_{0}=J_{f}^{-1 / n+1}$. The function $f$ will be holomorphic away from the zero set of $u_{0}$.

Proof. According to the previous lemma we can find $F=\vec{v} / v_{0}$ where $\left\{v_{0}, v_{1}, \ldots, v_{n}\right\}$ are a linearly independent solutions of the system (2.4) with $P_{i j}^{k}=S_{i j}^{k}$ and $v_{0}=$ $J_{F}^{-1 / n+1}$. As $u_{0}$ is solution of the system we have that $u_{0}=\alpha_{0} v_{0}+\cdots+\alpha_{n} v_{n}$. We need to find a Möbius mapping $T$ such that

$$
T \circ F=\left(\frac{u_{1}}{u_{0}}, \ldots, \frac{u_{n}}{u_{0}}\right)=f,
$$

and $J_{T \circ F}^{-1 / n+1}=u_{0}$. We have

$$
\begin{aligned}
J_{T \circ F}^{-1 / n+1}(z) & =J_{T}^{-1 / n+1}(F(z)) J_{F}^{-1 / n+1}(z) \\
& =\left(\lambda_{0}+\lambda_{1} F_{1}(z)+\cdots+\lambda_{n} F_{n}(z)\right) J_{F}^{-1 / n+1}(z) \\
& =\lambda_{0} v_{0}+\lambda_{1} v_{1}+\cdots+\lambda_{n} v_{n},
\end{aligned}
$$

which will be equal to $u_{0}$ if we choose $\lambda_{i}=\alpha_{i}$ for all $i=0,1, \ldots, n$. 


\section{Results}

Let $\Omega \subset \mathbf{C}^{n}$ be domain.

Theorem 3.1. Let $f: \Omega \rightarrow \mathbf{C}^{n}$ be a locally biholomorphic mapping. The following statements are equivalent:

(i) $S_{i j}^{0} f(z) \equiv 0$.

(ii) There exists a locally biholomorphic mapping $g: \Omega \rightarrow \mathbf{C}^{n}$ with $S_{g}=S_{f}$ and $J_{g}$ constant.

(iii) There exists a locally biholomorphic mapping $h: \Omega \rightarrow \mathbf{C}^{n}$ such that $S_{h}=S_{f}$ and $J_{h}^{-1 / n+1}=1 / L(h)$, where $L(w)=\alpha_{0}+\alpha_{1} w_{1}+\cdots+\alpha_{n} w_{n}$.

(iv) Locally there exists a biholomorphic change of variables such that the system (2.4) with $P_{i j}^{k}=S_{i j}^{k} f$ reduces to $\operatorname{Hess}(u)=0$.

Proof. (i) $\Rightarrow$ (ii). As $S_{i j}^{0} f \equiv 0$, the system (2.4) reduces to

$$
u_{i j}=\sum_{k=1}^{n} S_{i j}^{k} u_{k} .
$$

Therefore $u \equiv c$ is solution, thus by Lemma (2.3) there exists a function $g$ such that $J_{g} \equiv C$.

(ii) $\Rightarrow$ (iii). Let $g=T \circ h$ for some Möbius $T$ to be determined. Then $J_{g}^{-1 / n+1}(z)$ $=J_{T}^{-1 / n+1}(h(z)) J_{h}^{-1 / n+1}(z)$. Since $J_{g}^{-1 / n+1} \equiv C$, we have that

$$
C=\left(a_{0}+a_{1} h_{1}+\cdots+a_{n} h_{n}\right) J_{h}^{-1 / n+1}(z),
$$

from where the result obtains after scaling $h$.

(iii) $\Rightarrow$ (iv). Suppose $h$ has $J_{h}^{-1 / n+1}=1 / L(h)$. The previous argument shows that by choosing $T$ appropriately, we can produce $g=T \circ h$ with $J_{g} \equiv 1$. Hence $S_{g}(z)(\cdot, \cdot)=(D g(z))^{-1} D^{2} g(z)(\cdot, \cdot)$, and the system $(2.4)$ reduces to

$$
\operatorname{Hess} u(z)(\cdot, \cdot)=\nabla u(z) \cdot S_{g}(z)(\cdot, \cdot) .
$$

We consider $D\left(\nabla u(z)(D g(z))^{-1}\right)(\cdot, \cdot)$ :

$$
\begin{aligned}
D\left(\nabla u(z)(D g(z))^{-1}(\cdot, \cdot)=\right. & \operatorname{Hess} u(z)\left((D g(z))^{-1}(\cdot), \cdot\right) \\
& -\nabla u(z) \cdot(D g(z))^{-1} D^{2} g(z)\left((D g(z))^{-1}(\cdot), \cdot\right) \\
= & \nabla u(z) \cdot S_{g}(z)\left((D g(z))^{-1}(\cdot), \cdot\right) \\
& -\nabla u(z) \cdot(D g(z))^{-1} D^{2} g(z)\left((D g(z))^{-1}(\cdot), \cdot\right)=0 .
\end{aligned}
$$

Let $\varphi$ a local inverse of $g$. Therefore $U(w)=u(\varphi(w))$ satisfies that $\nabla U=\nabla u \cdot D \varphi=$ $\nabla u(z)(D g(z))^{-1}$, thus Hess $U(w) \equiv 0$.

(iv) $\Rightarrow(\mathrm{i})$. Since Hess $u(s) \equiv 0$, then $u \equiv c$ is a solution of this system (2.4), therefore $S_{i j}^{0} f \equiv 0$.

Theorem 3.2. Let $f: \Omega \rightarrow \mathbf{C}^{n}$ be a locally biholomorphic mapping. There exists a function $g: \Omega \rightarrow \mathbf{C}^{n}$ locally biholomorphic such that

$$
D g(z)=D f(z) J_{f}^{-\frac{2}{n+1}}
$$

if and only if $S_{i j}^{0} f \equiv 0$ for all $i$ and $j$. The function $g$ will have $S_{g}=S_{f}$. 
Proof. Suppose (3.1) holds. A straightforward calculation shows that

$$
(D g(z))^{-1} D^{2} g(z)(v, v)=S_{f}(z)(v, v) .
$$

The coordinate functions $g^{i}$ of function $g$ satisfy

$$
d g^{i}=J_{f}^{-2 / n+1} d f^{i} .
$$

Since $0=d^{2} g^{i}=d^{2} f^{i}$ we conclude that $J_{f}$ must be a constant. By Theorem 3.1 we conclude that $S_{i j}^{0} f \equiv 0$ for all $i$ and $j$. Reciprocally, if $S_{i j}^{0} f \equiv 0$, then there exists a constant solution of the system (2.4), and by Lemma 2.2 there exists a mapping $g$ with $S_{g}=S_{f}$ and $J_{g}^{-1 / n+1} \equiv C$. By $(2.5), S_{g}=P_{g}=S_{f}$.

Remark 3.3. Considering $S_{i j}^{0} f \equiv 0$ then $c D f=D g$ for some constant $c$. When $c=J_{f}^{-2 / n+1}$, we have that

$$
P_{g}(z)=S_{f}(z)=P_{f}(z) .
$$

Goldberg in [7] showed that, in terms of our operator,

$$
\operatorname{tr}\left\{D f(z)^{-1} D^{2} f(z)\left(\vec{v}_{i}, \cdot\right)\right\}=\frac{\partial}{\partial z_{i}} \log J_{f}(z),
$$

where $\vec{v}_{i}=(0, \ldots, 1, \ldots, 0)$ with 1 in position $i$. We use this result to prove the next theorem of uniqueness.

Theorem 3.4. Let $f, g$ be locally biholomorphic mappings defined in $\Omega$. Then $P_{f}(z)(\cdot, \cdot)=P_{g}(z)(\cdot, \cdot)$ if and only if $f=T \circ g$, where $T(z)=A z+b$ with $A$ is a $n \times n$ constant matrix and $b \in \mathbf{C}^{n}$.

Proof. Let $f$ and $g$ be locally biholomorphic mappings in $\Omega$. As $P_{f}(z)\left(\vec{v}_{i}, \cdot\right)=$ $P_{g}(z)\left(\vec{v}_{i}, \cdot\right)$ for all $i=1, \ldots, n$, then by equation $(3.2)$ we have that

$$
\nabla \log J_{f}(z)=\nabla \log J_{g}(z) .
$$

Using equation (2.5) we can conclude that $S_{f}(z)=S_{g}(z)$ for all $z$. Hence $g=T \circ f$ for some Mobius mapping T. But $\log J_{g}(z)=\log J_{T}(f(z))+\log J_{f}(z)$ and equation (3.3) we have that $\log J_{T}(z)$ is a constant, therefore $T(z)=A z+b$ for some $n \times n$ matrix $A$ and $b \in \mathbf{C}^{n}$. Reciprocally, if $f=T \circ g$ with $T(z)=A z+b$ for some $n \times n$ matrix $A$ and $b \in \mathbf{C}^{n}$, it is easy to see that $D f(z)=D T(f(z)) D f(z)=A D f(z)$, which implies that $P_{f}(z)=P_{g}(z)$.

Theorem 3.5. Let $A(z)$ be a bilinear operator defined in $\Omega$ by

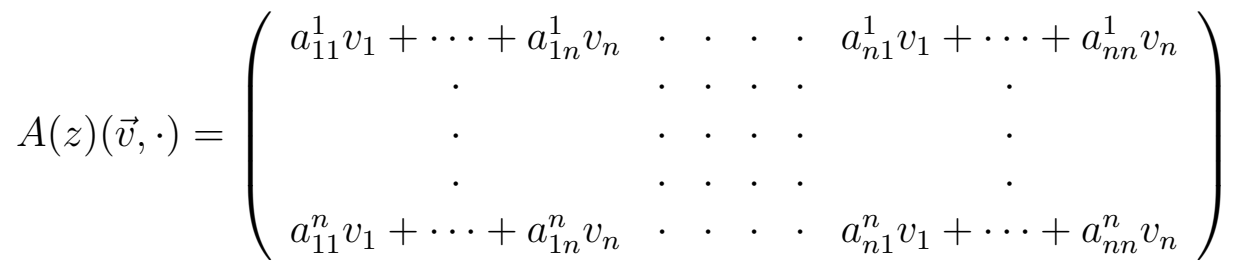

where $a_{i j}^{k}=a_{i j}^{k}(z)$ and $\vec{v}=\left(v_{1} \ldots, v_{n}\right)$. Then there exists a function $f: \Omega \rightarrow \mathbf{C}^{n}$ locally biholomorphic such that $P_{f}(z)=A(z)$ if and only if the following statements hold:

(i) $a_{i j}^{k}(z)=a_{j i}^{k}(z)$ for all $i, j, k=1, \ldots, n$; 
(ii) there exists a holomorphic function $\varphi: \Omega \rightarrow \mathbf{C}$ such that

$$
a_{1 j}^{1}(z)+a_{2 j}^{2}(z)+\cdots+a_{n j}^{n}(z)=\frac{\partial \varphi}{\partial z_{j}}(z) \forall j=1, \ldots, n ;
$$

(iii) $\exp \left(-\frac{\varphi}{n+1}\right)$ is a solution of the system (2.4) with $P_{i j}^{k}(z)$ given by

$$
P_{i j}^{k}(z)=a_{i j}^{k}(z)-\frac{1}{n+1}\left(\delta _ { i } ^ { k } \operatorname { t r } \left\{A(z)\left(\vec{v}_{j}, \cdot\right)+\delta_{j}^{k} \operatorname{tr}\left\{A(z)\left(\vec{v}_{i}, \cdot\right)\right),\right.\right.
$$

$i, j, k=1, \ldots, n$, and $P_{i j}^{0}(z)$ are defined in terms of $P_{i j}^{k}(z)$ such that the integrable condition of the system [25, pages 129-130] holds.

Proof. Using (i) and (ii) we have that

$$
\operatorname{tr}\{A(z)(\lambda, \cdot)\}=\nabla \varphi(z) \cdot \lambda
$$

For given $A(z)$ we can construct a bilinear mapping $\Lambda(z)(\lambda, \mu)$ as

$$
\Lambda(z)(\lambda, \mu)=A(z)(\lambda, \mu)-\frac{1}{n+1} \operatorname{tr}\{A(z)(\lambda, \cdot)\} \mu-\frac{1}{n+1} \operatorname{tr}\{A(z)(\mu, \cdot)\} \lambda .
$$

Each component of $\Lambda(z)$ is $P_{i j}^{k}$ defined by

$$
a_{i j}^{k}(z)-\frac{1}{n+1}\left(\delta _ { i } ^ { k } \operatorname { t r } \left\{A(z)\left(\vec{v}_{j}, \cdot\right)+\delta_{j}^{k} \operatorname{tr}\left\{A(z)\left(\vec{v}_{i}, \cdot\right)\right) .\right.\right.
$$

These coefficients satisfy $\sum_{i} P_{i k}^{k}=0$ for all $k=1, \ldots, n$. Now we define coefficients $P_{i j}^{0}$ in terms of $P_{i j}^{k}$ with $k=1, \ldots, n$ such that the integrability conditions in $[25$, pages 129-130] hold. Thus, the system (2.4) is completely integrable and in canonical form. Hence we can construct a function $f$ such that $S_{f}(z)=\Lambda(z)$. By (iii) we have that

$$
J_{f}^{-1 / n+1}=\exp \left(-\frac{\varphi}{n+1}\right)
$$

As $S_{f}$ is defined by equation $(2.5)$ we conclude that

$$
\operatorname{tr}\{A(z)(\lambda, \cdot)\}=\frac{1}{n+1} \nabla J_{f}(z) \cdot \lambda,
$$

which implies that

$$
P_{f}(z)=(D f(z))^{-1} D^{2} f(z)(\cdot, \cdot)=A(z)(\cdot, \cdot) .
$$

Reciprocally, it is easy to see that $P_{f}(z)$ satisfies (i), (ii) and (iii).

Observe that $\alpha[D f(z)]^{-1} D^{2} f(z)(\vec{v}, \cdot)$ for a locally biholomorphic function $f$ satisfies (i), (ii) and (iii) of Theorem 3.4.

Definition 3.6. Let $f$ be a locally biholomorphic mapping in $\Omega$ such that $f(0)=$ 0 and $D f(0)=$ Id. We define $f_{\alpha}$ in $\Omega$ as the locally biholomorphic mapping for which

$$
\left[D f_{\alpha}(z)\right]^{-1} D^{2} f_{\alpha}(z)(\cdot, \cdot)=\alpha[D f(z)]^{-1} D^{2} f(z)(\cdot, \cdot),
$$

and $f_{\alpha}(0)=0, D f_{\alpha}(0)=\mathrm{Id}$.

As a generalization of the problem raised in [6], one can ask the question of determining the values of $\alpha$ for which the mapping $f_{\alpha}$ is univalent when $f$ is univalent or even just locally univalent. A partial answer is given below when $f$ is convex in the unit ball $\mathbf{B}^{n}$. Theorem 3.5 shows another partial result for compact linear invariant families. Since the class of univalent mappings in $\mathbf{B}^{n}$ fails to be compact $(n>1)$, we 
think it is unlikely that there exists an $\alpha_{0}>0$ small enough so that $f_{\alpha}$ is univalent for any $|\alpha| \leq \alpha_{0}$ and $f$ univalent in $\mathbf{B}^{n}$. An interesting compact family of univalent mappings to consider would be the class $S_{0}$ of univalent mappings in $\mathbf{B}^{n}$ that have a parametric representation.

Example 3.7. Let $f\left(z_{1}, z_{2}\right)=\left(\phi_{\alpha}\left(z_{1}\right), \psi_{\alpha}\left(z_{2}\right)\right)$ be a locally univalent mapping defined in $\mathbf{B}^{2}$ such that $\phi_{\alpha}\left(z_{1}\right)$ and $\psi_{\alpha}\left(z_{2}\right)$ are defined by the equation (1.1) where $\phi$ and $\psi$ are locally univalent analytic mappings defined in the unit disc such that $\phi(0)=\psi(0)=0, \phi^{\prime}(0)=\psi^{\prime}(0)=1$ and suppose that $z=\left(z_{1}, z_{2}\right) \in \mathbf{B}^{2}$. Its Schwarzian derivatives satisfy

$$
\begin{aligned}
& S_{11}^{1} f\left(z_{1}, z_{2}\right)=\frac{\phi_{\alpha}^{\prime \prime}}{\phi_{\alpha}^{\prime}}\left(z_{1}\right)=\alpha \frac{\phi^{\prime \prime}}{\phi^{\prime}}\left(z_{1}\right), \quad S_{22}^{2} f\left(z_{1}, z_{2}\right)=\frac{\psi_{\alpha}^{\prime \prime}}{\psi_{\alpha}^{\prime}}\left(z_{2}\right)=\alpha \frac{\psi^{\prime \prime}}{\psi^{\prime}}\left(z_{2}\right), \\
& S_{22}^{1} f\left(z_{1}, z_{2}\right)=S_{11}^{2} f\left(z_{1}, z_{2}\right)=0 .
\end{aligned}
$$

Now, let $f(z)=\left(\psi\left(z_{1}\right), \phi\left(z_{2}\right)\right)$. Then the corresponding mapping $f_{\alpha}$ has the property that its Schwarzian derivatives are

$$
\begin{aligned}
& S_{11}^{1} f_{\alpha}\left(z_{1}, z_{2}\right)=\alpha S_{11}^{1} f\left(z_{1}, z_{2}\right)=\alpha \frac{\phi^{\prime \prime}}{\phi^{\prime}}\left(z_{1}\right), \\
& S_{22}^{2} f_{\alpha}\left(z_{1}, z_{2}\right)=\alpha S_{22}^{2} f\left(z_{1}, z_{2}\right)=\alpha \frac{\psi^{\prime \prime}}{\psi^{\prime}}\left(z_{2}\right), \\
& S_{22}^{1} f_{\alpha}\left(z_{1}, z_{2}\right)=S_{11}^{2} f_{\alpha}\left(z_{1}, z_{2}\right)=0 .
\end{aligned}
$$

Therefore $S_{i j}^{k} f=S_{i j}^{k} f_{\alpha}$ which implies that there exists a Möbius mapping $M$ such that $M \circ f=f_{\alpha}$. But $f(0)=0=f_{\alpha}(0), D F(0)=I d=D f_{\alpha}(0)$ and $\nabla \log J_{f}=$ $\nabla \log J_{f_{\alpha}}=\alpha \nabla \log J_{f}$, then $f=f_{\alpha}$. Thus

$$
f(z)=\left(\phi\left(z_{1}\right), \psi\left(z_{2}\right)\right) \Longrightarrow f_{\alpha}(z)=\left(\phi_{\alpha}\left(z_{1}\right), \psi_{\alpha}\left(z_{2}\right)\right),
$$

where $\phi_{\alpha}$ and $\psi_{\alpha}$ are defined by (1.1). By the way, in this example, if $|\alpha|<1 / 4$, then $f_{\alpha}$ will be univalent in $\mathbf{B}^{2}$. Moreover, if $\phi\left(z_{1}\right)$ is a univalent mapping defined by $(1.2)$ and $\psi\left(z_{2}\right)=z_{2}$, then the mapping $f(z)=\left(\phi\left(z_{1}\right), \psi\left(z_{2}\right)\right)$ is univalent and the corresponding mapping $f_{\alpha}$ is not univalent if $|\alpha|>1 / 3$ and $\alpha \neq 1$.

In [9] the author proved that a locally biholomorphic mapping $f: \mathbf{B}^{n} \rightarrow \mathbf{C}^{n}$ is convex if and only if $1-\operatorname{Re}\left\langle[D f(z)]^{-1} D^{2} f(z)(u, u), z\right\rangle>0$ for all $z \in \mathbf{B}^{n}$ and $u \in \mathbf{C}^{n}$ with $\|u\|=1$. Thus, if $0 \leq \alpha \leq 1$, then $f_{\alpha}$ is a convex mapping when $f$ is a convex mapping since

$$
1-\operatorname{Re}\left\langle\left[D f_{\alpha}(z)\right]^{-1} D^{2} f_{\alpha}(z)(u, u), z\right\rangle=1-\alpha \operatorname{Re}\left\langle[D f(z)]^{-1} D^{2} f(z)(u, u), z\right\rangle>0
$$

Example 3.8. Let $f$ be a univalent function in D. We consider the RoperSuffridge extension (see [21]) to $\mathbf{B}^{2}$ of $f$ to the function

$$
\Phi_{f}(z)=\left(f\left(z_{1}\right), \sqrt{f^{\prime}\left(z_{1}\right)} z_{2}\right)
$$

Thus,

$$
\left[D \Phi_{f}(z)\right]^{-1}\left[D^{2} \Phi_{f}(z)\right](\vec{v}, \cdot)=\left(\begin{array}{cc}
\frac{f^{\prime \prime}}{f^{\prime}}\left(z_{1}\right) v_{1} & 0 \\
\frac{1}{2} z_{2} S f\left(z_{1}\right) v_{1}+\frac{1}{2} \frac{f^{\prime \prime}}{f^{\prime}}\left(z_{1}\right) v_{2} & \frac{1}{2} \frac{f^{\prime \prime}}{f^{\prime}}\left(z_{1}\right) v_{1}
\end{array}\right) .
$$

A straightforward calculation shows that

$$
\left(\Phi_{f}\right)_{\alpha}(z)=\left(f_{\alpha}\left(z_{1}\right), z_{2} \sqrt{f_{\alpha}^{\prime}\left(z_{1}\right)}+y\left(z_{1}\right)\right)
$$


where $f_{\alpha}$ is defined by equation (1.1) and $y$ satisfies that

$$
y^{\prime \prime}-\alpha \frac{f^{\prime \prime}}{f^{\prime}} y^{\prime}=\frac{\alpha(\alpha-1)}{4}\left(\frac{f^{\prime \prime}}{f^{\prime}}\right)^{2}\left(f^{\prime}\right)^{\alpha / 2} .
$$

Moreover, $\Phi_{f}$ is univalent when $f$ is univalent, in fact, if $f$ is convex, then $\Phi_{f}$ is convex. On the other hand, $\left(\Phi_{f}\right)_{\alpha}$ is univalent if $f_{\alpha}$ is univalent which holds for $|\alpha| \leq 1 / 4$ for all univalent mappings $f$.

Theorem 3.9. Let $f: \mathbf{B}^{n} \rightarrow \mathbf{C}^{n}$ be a locally biholomorphic mapping such that the norm order of the linear invariant family generated by $f$ is $\beta<\infty$. Then $f_{\alpha}$ is univalent if $|\alpha| \leq \frac{1}{2 \beta+1}$.

Proof. Let $\phi$ be a automorphism of $\mathbf{B}^{n}$ such that $\phi(0)=\zeta$. The mapping $g(z)=D \phi(0)^{-1} D f(\phi(0))^{-1}(f(\phi(z))-f(\phi(0)))$ belongs to the family generated by $f$, therefore $\left\|D^{2} g(0)\right\| \leq \beta$. But

$$
\begin{aligned}
D^{2} g(0)(\cdot, \cdot)= & D \phi(0)^{-1} D f(\zeta)^{-1} D f(w) D^{2} \phi(0)(\cdot, \cdot) \\
& +D \phi(0)^{-1} D f(\zeta)^{-1} D^{2} f(\zeta)(D \phi(0)(\cdot), D \phi(0)(\cdot)) .
\end{aligned}
$$

Evaluating in $D \phi(0)^{-1}(\zeta)=\zeta /\left(1-\|\zeta\|^{2}\right)$, multiplication by $\alpha$ and using (3.4) we have that

$$
\begin{aligned}
\alpha D^{2} g(0)(\zeta, \cdot)= & \alpha D \phi(0)^{-1} D f(\zeta)^{-1} D f(\zeta) D^{2} \phi(0)(\zeta, \cdot) \\
& +\left(1-\|\zeta\|^{2}\right) D \phi(0)^{-1} D f_{\alpha}(\zeta)^{-1} D^{2} f_{\alpha}(\zeta)(\zeta, D \phi(0)(\cdot))
\end{aligned}
$$

where $D \phi(0)^{-1} D^{2} \phi(\zeta, \cdot)=-\|\zeta\|^{2}(\cdot)-\zeta \zeta^{*}(\cdot)$. Thus, for all vectors $v=D \phi(0)^{-1}(u)$ it follows that

$$
\left(1-\|\zeta\|^{2}\right) D f_{\alpha}(\zeta)^{-1} D^{2} f_{\alpha}(\zeta)(\zeta, u)=\alpha D \phi(0) D^{2} g(0)(\zeta, v)-\alpha\|\zeta\|^{2} u-\alpha\left(1-\|\zeta\|^{2}\right) \zeta \zeta^{*} v .
$$

Then taking the supremum over all vectors $u$ with norm $\|u\|=1$, we have that

$$
\left\|\left(1-\|\zeta\|^{2}\right) D f_{\alpha}(\zeta)^{-1} D^{2} f_{\alpha}(\zeta)(\zeta, \cdot)+\alpha\right\| \zeta\left\|^{2} I\right\| \leq|\alpha|(2 \beta+1) .
$$

Hence by the generalization of the Ahlfors and Becker result (see [10, page 350]) we conclude that $f_{\alpha}$ satisfies the hypothesis of this theorem, so it is univalent in $\mathbf{B}^{n}$.

The last corollary is an immediate consequence.

Corollary 3.10. Let $\mathscr{F}$ be a linearly invariant family of locally biholomorphic mappings defined in $\mathbf{B}^{n}$ of finite order $\beta$. Then $f_{\alpha}$ is univalent in $\mathbf{B}^{n}$ for every $f \in \mathscr{F}$ when $|\alpha| \leq \frac{1}{2 \beta+1}$.

Acknowledgements. I would like to thank Martin Chuaqui and John Pfaltzgraff for their useful suggestions and valuable discussions.

\section{References}

[1] Ahlfors, L. V.: Sufficient conditions for quasi-conformal extension. - In: Discontinuous groups and Riemann surfaces (Proc. Conf., Univ. Maryland, College Park, Md., 1973), Ann. of Math. Studies 79, 1974, 23-29.

[2] BeCKer, J.: Löwnersche Differentialgleichung und quasi-konform forttsetzbare schlichte Funktionen. - J. Reine Angew. Math. 225, 1972, 23-43. 
[3] Chunqui, M., and B. Osgood: Sharp distortion theorems associated with the Schwarzian derivative. - J. London Math. Soc. (2) 48, 1993, 289-298.

[4] Chunqui, M., and B. Osgood: Ahlfors-Weill extensions of conformal mappings and critical points of the Poincaré metric. - Comment. Math. Helv. 69, 1994, 659-668.

[5] Duren, P.: Univalent functions. - Springer-Verlag, 1983.

[6] Duren, P., H.S. Shapiro, and A. L. Shields: Singular measures and domains not of Smirnov type. - Duke Math. J. 33:2, 1966, 247-254.

[7] Goldberg, M. A.: The derivative of a determinant. - Amer. Math. Monthly, 79, 1972, 11241126.

[8] Kim, Y. J., and E. P. Merkes: On an integral of poers of spirallike function. - Kyungpook Math. J. 12, 1972, 249-253.

[9] Kikuchi, K.: Starlike and convex mappings in several complex variables. - Pacif. J. Math. 44, 1973, 569-580.

[10] Graham, I., and G. Kohr: Geometric function theory in one and higher dimensions. Monogr. Textbooks Pure Appl. Math. 255, Marcel Dekker, 2003.

[11] Hernández, R.: Schwarzian derivatives and a linearly invariant family in $\mathbf{C}^{n}$. - Pacific J. Math. 228:2, 2006, 201-218.

[12] Hernández, R.: Schwarzian derivatives and some criteria for univalence in $\mathbf{C}^{n}$. - Complex Var. Elliptic Equ. 52:5, 2007, 397-410.

[13] Molzon, R., and K. P. Mortensen: Univalence of holomorphic mappings. - Pacific J. Math. 180:1, 1997, 125-133.

[14] Molzon, R., and H. TAmanor: Generalized Schwarzians in several variables and Möbius invariant differential operators. - Forum Math. 14, 2002, 165-188.

[15] Nehari, Z.: The Schwarzian derivative and schlicht functions. - Bull. Amer. Math. Soc. 55, $1949,545-551$

[16] Nehari, Z.: Some criteria of univalence. - Proc. Amer. Math. Soc. 5, 1954, 700-704.

[17] ODA, T.: On Schwarzian derivatives in several variables. - Kokyuroku of R.I.M. 226, Kioto Univ., 1974 (in Japanese).

[18] Pfaltzgraff, J. A.: Subordination chains and univalence of holomorphic mappings in $\mathbf{C}^{n}$. Math. Ann. 210, 1974, 55-68.

[19] Pfaltzgraff, J. A.: Univalence of the integral of $f^{\prime}(z)^{\lambda}$. - Bull. London Math. Soc. 7, 1975, $254-256$.

[20] Pommerenke, Ch.: Linear-invarinte Familien analytischer Funktionen I. - Math. Ann. 155, 1964, 108-154.

[21] Roper, K., and T. Suffridge: Convex mappings on the unit ball in $\mathbf{C}^{n}$. - J. Anal. Math. 65, 1995, 333-347.

[22] Suffridge, T.: Starlikeness, convexity and other geometric properties of holomorphic maps in higher dimensions. - In: Complex analysis (Proc. Conf., Univ. Kentucky, Lexington, Ky., 1976), Lecture Notes in Math. 599, Springer-Verlag, New York, 1976, 146-159.

[23] Royster, W. C.: On the univalence of a certain integral. - Michigan Math. J. 12, 1965, $385-387$.

[24] Yoshida, M.: Canonical forms of some system of linear partial differential equations. - Proc. Japan Acad. 52, 1976, 473-476.

[25] YoshidA, M.: Orbifold-uniformizing differential equations. - Math. Ann. 267, 1984, 125-142. 